Sir,

\section{Errors in self-programming the Hoffer $Q$ formula}

I was surprised to read the article entitled, 'Comparison of IOL power calculations by the IOLMaster $v s$ theoretical calculations' (Eye 2006; 20: 90-97) by S Basu. Instead of using an officially approved, commercially available computer program (Holladay ${ }^{\circledR}$ IOL Consultant, Hoffer $^{\circledR}$ Programs, etc) to act as the gold standard to test the accuracy of the IOLMaster calculation, the authors chose to create their own computer program ('custommade computer program') to do so. Also, they never asked the authors of the formulas to validate that their personal computerization was done correctly and accurately.

After evaluating their computer program data at the end of their paper, it appears that the authors did set the proper index of refraction (1.3375) for the Hoffer $Q$ to work correctly in the IOLMaster.

The original article ${ }^{1}$ for the Hoffer $Q$ formula contained both typographical errors and formula changes, which were attempted to be corrected by a published erratum ${ }^{2}$ in an issue that appeared several months later. Because of the nearness to the end of the year, the erratum was published the next year (1994) and in the next volume (20).

Those who have used the original article to program the formula had no way of knowing that the corrections were published in a later issue.

There are five specific issues regarding the original publication and its erratum:

- The first is that an important and crucial minus sign was left out of the power formula $(P)$ as corrected in the erratum.

- The second is that the expression ' 1,000 ' in the power formula $(P)$ has been misinterpreted by some Europeans because of the comma. It should have been expressed as ' 1000 ' instead.

- The third is that in the example calculations for the 'Hyperopic eye (Example 2)'; the results for Emmetropia and Ametropia are switched as described in the erratum.

- The fourth was a change that I made in the parameters to calculate the $\mathrm{Q}$ formula for the ACD. The original article placed limits on the ACD. This was replaced with limits on the axial length (AL) (see below). Unfortunately, the erratum did not stress that this was a replacement rather than just an addition, so many have used both the limitation on the AL as well as that on the ACD leading to errors in calculation.

- The fifth is an error that has often occurred. There are two limitations placed on the AL. The first is: If $\mathrm{AL} \leqslant 23: M=+1$ and $G=28.0$; if $\mathrm{AL}>23: M=-1$ and $G=23.5$ noted in the original article. The second is that which was added in the erratum: If $\mathrm{AL}>31: \mathrm{AL}=31.0$; if $\mathrm{AL}<18.5: \mathrm{AL}=18.5$. Both of these $\mathrm{AL}$ limitations ONLY apply to the $\mathrm{Q}$ part of the formula to calculate $\mathrm{ELP}:\left[\mathrm{ACD}=\mathrm{pACD}+0.3(\mathrm{AL}-23.5)+(\tan K)^{2}+(0.1 * M\right.$ $\left.(23.5-\mathrm{AL})^{2} *\left(\tan \left(0.1(G-\mathrm{AL})^{2}\right)\right)-0.99166\right]$

They do not apply to the AL used in the power formula. $[P=(1336 /(\mathrm{AL}-\mathrm{ACD}-0.05))-(1.336 /((1.336 /(K+R))-$ $((\mathrm{ACD}+0.05) / 1000)))$.

The paper by Basu appears to include the limitations on the $\mathrm{ACD}$ [If $\mathrm{ACD}>6.5, \mathrm{ACD}=6.5$ : If $\mathrm{ACD}<2.5$, $\mathrm{ACD}=2.5$ ], which they should not have done. The factor [If $\mathrm{AL}>31, \mathrm{AL}=31$; If $\mathrm{AL}<16.5, \mathrm{AL}=16.5$ ] replaced the limits on the ACD.

I also cannot tell from reading their code whether they took into account the missing minus (-) sign between the '...R $)$...' and the '...((C...' in the original paper.

Did they use a retinal thickness factor with the Hoffer Q? The Hoffer Q does not use one.

All the multiple errors by the publisher and in the later errata have made programming the Hoffer $Q$ formula fraught with great danger. Why did the authors not consider checking their computer program with the formula authors, or use an approved and licensed computer program before publishing something so blatantly detrimental to the Zeiss IOLMaster or Hoffer Q formula, both of which are perfectly correct?

In this age of technology, time zones and the Atlantic Ocean are no excuse. It is now time for $\mathrm{S}$ Basu to publish an erratum.

\section{References}

1 Hoffer KJ. The Hoffer Q formula: a comparison of theoretic and regression formulas. J Cataract Refract Surg 1993; 19: 700-712.

2 Erratum. J Cataract Refract Surg 1994; 20: 677.

KJ Hoffer

University of California, Los Angeles

1301 20th Street \#250, Santa Monica, CA 90404, USA

Correspondence: KJ Hoffer,

Tel: + 1310451 2020;

Fax: + 13103955947

E-mail: khoffermd@aol.com

Eye (2007) 21, 429. doi:10.1038/sj.eye.6702559; published online 29 September 2006 